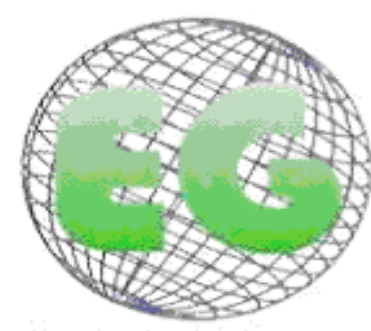

ISSN 1695-6141 $\mathrm{N}^{\circ} 27$
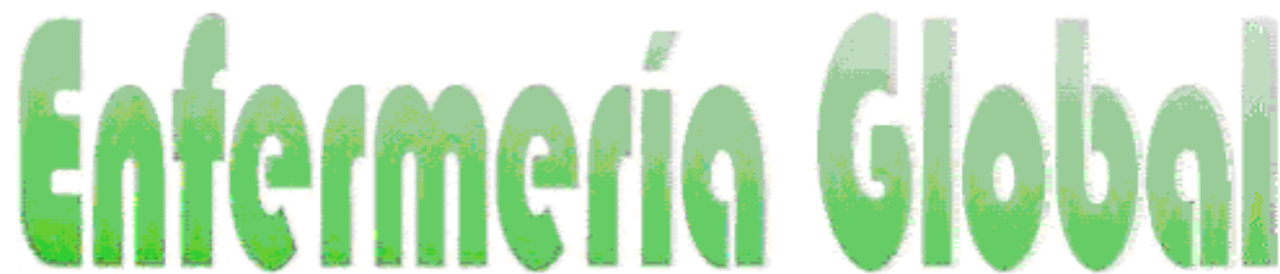

Revista electrónica trimestral de Enfermería

Julio 2012

www.um.es/egloball

ENFERVERIA Y PERSPECTIVA DE GÉNERO

\title{
Frecuencia de la depresión puerperal en la maternidad de un hospital universitario de la Región del Sur
}

Frequência da depressão puerperal na maternidade de um hospital universitário da Região Sul

\section{*Lopes Menezes, F., "*Netto de Oliveira, AM., ${ }^{* * *}$ Pinto Lemos, LA., ${ }^{* * * *}$ Arruda da} Silva, P., "**Pereira da Costa Kerber, N., **Santos da Silva, MR.

\author{
*Enfermera. E- mail: francislenelm@yahoo.com.br ${ }^{* *}$ Doctora en Enfermería. Docente de la Escuela de \\ Enfermería. ${ }^{* * *}$ Profesor Titular del Instituto de Matemáticas, Estadística y Física Aplicada de la Escuela de \\ Enfermería ****Doctoranda en Enfermería. Universidad Federal de Rio Grande. Brasil.
}

\begin{abstract}
Palabras clave: depresión posparto; enfermería; salud de la mujer.
Palavras-chave: depressão pós-parto; enfermagem; saúde da mulher.

Keywords: Postpartum Depression; nursing; women's health
\end{abstract}

\section{RESUMEN}

Este estudio tuvo como objetivo identificar la frecuencia de riesgo para desencadenar la depresión posparto, en puérperas internadas en la maternidad de un Hospital Universitario, a través de la aplicación de la escala Edinburgh Post-Natal Depression Scale (EPDS).

Se trata de un estudio cuantitativo, exploratorio descriptivo. Participaran del estudio 53 puérperas en posparto inmediato en el período de abril a mayo de 2009. Fue aplicado un cuestionario elaborado con base en la escala EPDS.

Los datos obtenidos a través de la aplicación de la escala fueran analizados e interpretados, a través de la estadística descriptiva. Se respetaron todas las determinaciones de la Resolución 196/96 de estudio con seres humanos.

Los resultados señalaron que el riesgo de depresión posparto fue encontrado en seis puérperas (11\%), lo que demuestra la importancia de los profesionales de la salud en realizar la detección precoz de la depresión posparto, teniendo como ayuda la escala EPDS, por la eficacia y practicidad de su aplicación.

\section{RESUMO}

Este estudo teve como objetivo identificar a freqüência de risco para desencadear a depressão pós-parto, em puérperas internadas na maternidade de um Hospital Universitário, através da aplicação da escala Edinburgh Post-Natal Depression Scale (EPDS). 
Trata-se de um estudo quantitativo, exploratório descritivo. Participaram do estudo 53 puérperas em pós-parto imediato no período de abril à maio de 2009. Foi aplicado um questionário elaborado com base na escala Edinbugh Post-Natal Depression Scale (EPDS).

Os dados obtidos através da aplicação da escala foram analisados e interpretados, através da estatística descritiva. Foram respeitadas todas as determinações da Resolução 196/96 de pesquisa com seres humanos.

Os resultados apontaram que o risco de depressão pós-parto foi encontrado em seis puérperas (11\%), o que demonstra a importância dos profissionais da saúde em realizar a detecção precoce da depressão pós-parto, tendo como auxílio à escala EPDS, pela eficácia e praticidade da sua aplicação.

\section{ABSTRACT}

This study aimed to identify the frequency of risk to trigger postpartum depression in women interned in a Maternity Hospital, through the application of the scale Edinburgh Post-Natal Depression Scale (EPDS).

It is a quantitative, exploratory and descriptive study. It included 53 postpartum women in the immediate postpartum period from April to May 2009. A questionnaire was developed based on the scale Edinburgh PostNatal Depression Scale (EPDS).

The obtained data through the application of the scale were analyzed and interpreted using descriptive statistics and taking into account all the provisions from the Resolution196/96 of the research with humans.

The results showed that the risk of postpartum depression was found in six women (11\%), which demonstrate the importance of health professionals to perform the early detection of postpartum depression, with the EPDS scale aid, the effectiveness' and practicality of its implementation.

\section{INTRODUCCIÓN}

La depresión puerperal es considerada un trastorno mental que presenta una continuidad de respuestas emocionales después del parto, con niveles de manifestación significantes que interfieren en la calidad de vida de la madre y del niño. La psicosis posparto es la manifestación más grave de la enfermedad, pudiendo llevar a la madre a agredir a su hijo y/o practicar el infanticidio. Si la mujer presenta depresión posparto (DPP), no procura la ayuda de los profesionales de la salud y no recibe apoyo familiar, su estado puede agravarse hasta el punto de que ocurran situaciones caóticas en la vida de la familia ${ }^{1}$. La DPP, actualmente se encuentra incluida como un grave problema de salud pública.

La proyección de mujeres con depresión posparto se constituye en un elemento significativo a ser utilizado por los servicios de salud, en especial los enfermeros, en el sentido de reducir los índices de depresión puerperal. Así, para evaluar la salud de la puérpera, específicamente, en lo que se refiere a la detección de síntomas depresivos, son utilizadas escalas que describen el rastreo de la depresión posparto. Hasta donde sabemos no existen escalas diseñadas específicamente para la detección de depresión posparto durante la gravidez, aunque autores han utilizado la escala Edinburgh Post-Natal Depression Scale (EPDS) con esa finalidad ${ }^{2-3}$.

Traducida al portugués y validada en Brasil, la Edinburgh Post-Natal Depression Scale además detectar temprano la depresión, a través de una intervención efectiva y eficaz por parte de los profesionales, también evita posibles daños de la enfermedad sobre la madre, en la relación con su compañero, en la formación del vínculo con el niño y en el funcionamiento familiar. Los tratamientos más utilizados en la depresión puerperal incluyen las psicoterapias y/o psicofarmacoterapia ${ }^{4}$. 
La Escala de Edinburgh Post-Natal Depression Scale es compuesta por diez enunciados, mostrando sensibilidad y especificidad satisfactorias para el posible diagnóstico. Sus opciones son puntuadas de cero a tres, componiendo un total máximo de treinta puntos y en el mínimo cero, respectivamente. Se suman los números correspondientes a cada artículo, se obtiene la sensibilidad de la escala, con resultado mayor o igual a 11/12. Las mujeres que tienen el indicador umbral deben tener acompañamiento de un profesional de salud ${ }^{5}$.

Las estadísticas mundiales indican que cerca del 15\% de las puérperas tienen depresión posparto. En la población de baja renta, ese valor puede alacanzar el $25 \%{ }^{2}$. Respecto a la preocupación relativa al desempeño de las (los) enfermeras (os) como teniendo un papel fundamental mediante la detección precoz de la DPP, en función del vínculo que establecen con las puérperas, se entiende que la relación terapéutica facilita la verbalización de los pensamientos y sentimientos vividos por la mujer, en esta fase del ciclo vital, proporcionando la promoción de la salud mental, a través de la ayuda que este y otros profesionales de la salud pueden dar a estas.

Teniendo en cuenta que el conocimiento de la DPP es indispensable a las (a los) enfermeras (os), que actúan en el área obstétrica y pediátrica, al prestar cuidados directos a las puérperas y sus familiares, es imprescindible que se piense en identificar la inestabilidad y/o labilidad emocional de estas y dirigir las acciones de enfermería, en el sentido de ayudar a esas personas a enfrentar y superar las dificultades encontradas en este momento de transición del ciclo vital. Y, a partir de esos conocimientos prestar una asistencia de enfermería más cualificada y efectiva. En ese sentido el presente estudio tiene como objetivo Identificar la frecuencia de riesgo para desencadenar la depresión posparto, en puérperas internadas en la maternidad de un Hospital Universitario de la región Sur, a través de la aplicación de la escala Edinburgh Post-Natal Depression Scale (EPDS).

\section{METODOLOGÍA}

Se trata de una investigación cuantitativa, descriptiva exploratoria, fundamentada en datos obtenidos a través de la realización de un cuestionario elaborado con base en la escala Edinbugh Post-Natal Depression Scale (EPDS). Participaron del estudio 53 puérperas internadas en la maternidad de un Hospital Universitario de la región Sur del país, la cual es de referencia para la atención de partos de alto riesgo y gestantes portadoras de HIV, en la región sur del Estado de Rio Grande do Sul. La investigación se realizó en el período de abril a mayo de 2009. Se observó que, de media se realizan mensualmente 150 partos; con una significancia del $10 \%$ fue establecido un tamaño mínimo de muestra de 53 entrevistas, considerando la utilización de la EPDS.

Se invitó a participar del estudio a todas las puérperas que estaban internadas en la maternidad, en el período referido arriba. Se utilizaron como criterios para inclusión: puérperas en el segundo o tercero día después del parto y que aceparon participar de la investigación, y como criterios de exclusión: las puérperas con pérdida fetal, que estuviesen bajo tratamiento psicológico o psiquiátrico y las que se recusaran a participar.

El empleo de la escala EPDS tuvo como finalidad hacer el rastreo de las mujeres con mayor probabilidad para desencadenar depresión puerperal, determinando la frecuencia de riesgo para el desencadenamiento de la enfermedad. Este es un instrumento de auto-evaluación que contiene diez preguntas, con cuatro opciones que son puntuadas de 0 a 3 , de acuerdo con la presencia o intensidad de los síntomas: humor deprimido o disfórico, insomnio, pérdida del placer, disminución del desempeño en el día a día, sentimientos de culpa e ideas de muerte y suicidio. 
Las puérperas del estudio se insertaron en grupo de riesgo para desarrollar depresión, cuando los marcadores alcanzados en la EPDS fueran iguales o mayores que 11. Este punto de corte se fundamentó en estudio de validación realizado en Brasil, que demostró con marcador $11 / 12$, sensibilidad de $72 \%$, especificidad de $88 \%$, valor predictivo de $78 \%$ y eficiencia global de $83 \%$ para la correcta identificación de mujeres deprimidas ${ }^{6}$.

Atendiendo la posibilidad de identificar tempranamente mujeres con riesgo de desarrollar DPP o que ya se muestren deprimidas, el estudio propuesto aplica el cuestionario después del parto, cuando la paciente aun se encuentra en la maternidad, permitiendo la intervención secundaria rápida y minimizando las consecuencias de la enfermedad. Luego, se comparan las pacientes y las variables maternas (edad, número de gestaciones y tipo de parto), determinándose dos grupos de puérperas: las que obtuvieran marcadores $<11$ serían del grupo control (sin riesgo); y las con marcadores $\geq 11$ del grupo caso (de riesgo) para desarrollar la depresión pos-parto. Los casos que tenían el marcador umbral de diagnóstico probable de depresión posparto era comunicado a la enfermera encargada, a fin de que esta solicitase la evaluación del Servicio de Psicología de la institución.

Los datos fueran poscodificados, tabulados y recibieran el tratamiento estadístico del tipo descriptivo, con ayuda del Software Microsoft Office Excel (versión 2007) con el objetivo de construir una base de datos para análisis y así procesar y almacenar las respuestas en forma tabular. También se utilizaron conceptos de la estadística descriptiva (frecuencia de ocurrencia) y de análisis exploratorio de datos aplicáandose el Software Estadística 6.0, el cual contribuyó a ampliar las informaciones obtenidas, a partir de los datos, derivados de los cuestionarios aplicados a las puérperas, los cuales permitieron construir las tablas y los gráficos que facilitaron la visualización, descripción, comprensión y análisis de la frecuencia de ocurrencia de las variables del estudio.

Todas las participantes incluidas en esta pesquisa fueran informadas sobre el estudio y su carácter privado; estuvieron de acuerdo en participar y pusieron su nombre en el término de consentimiento libre y esclarecido. El estudio tuvo la aprobación del Comité de Ética y Pesquisa del Área de Salud (CEPAS), de la Universidad Federal do Rio Grande (FURG), bajo $\mathrm{n}^{\circ}$ de parecer 011/2009.

\section{RESULTADOS Y DISCUSIÓN}

Participaron 53 mujeres, las edades variaron entre 14 y 40 años, con media de edad de 26 años (mediana $=26$, desvío padrón $=7$ ), siendo 11 mujeres $(21 \%)$ de la muestra adolescentes, considerando la clasificación de la Organización Mundial de la Salud (OMS) que determina la adolescencia de los 10 a los 19 años (Gráficos 1 y 2). 
Gráfico 1: Distribución de la edad de las puérperas, entrevistadas en la Maternidad del Hospital Universitario de la FURG-2009.

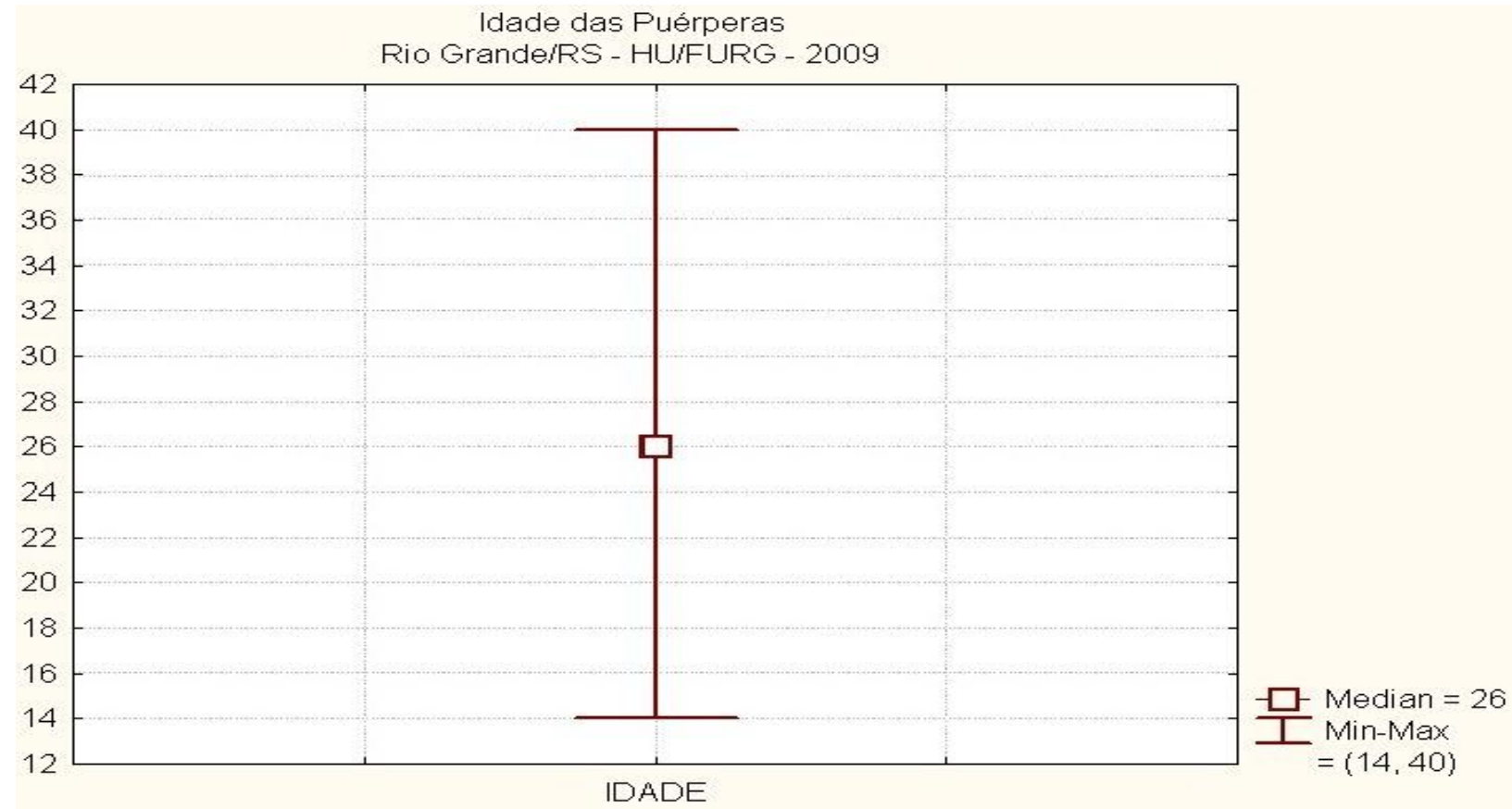

Gráfico 2: Histograma de la edad de las puérperas, entrevistadas en la Maternidad de un Hospital Universitario de la FURG- 2009.

Idade das Puérperas

Rio Grande/RS - HU/FURG - 2009

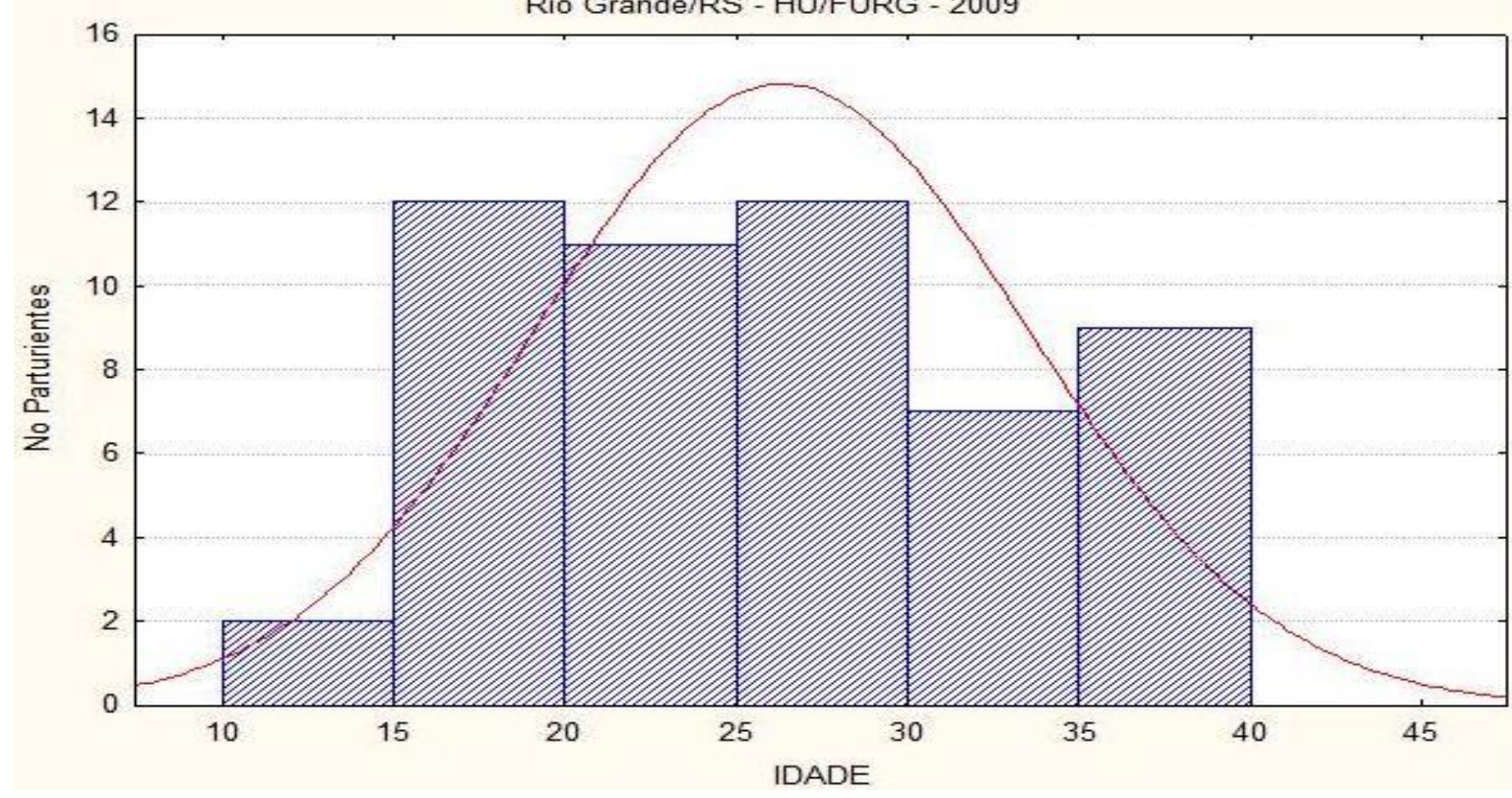

En relación a la puntuación de la EPDS, la mediana fue de 4 (Gráfico 3). Seis puérperas tuvieran marcadores $\geq 11$ de la EPDS, lo que representó $11 \%$ de la muestra con probabilidad de desarrollar la DPP; 47 (89\%) con marcadores inferiores a 11, las cuales fueran consideradas sin señales y síntomas depresivos (Gráfico 4). 
Gráfico 3: Distribución de puntuación de corte puérperas, según escala de Edinburgh.

Pontuação do EPDS

HUIFURG -Rio Grande - 2009

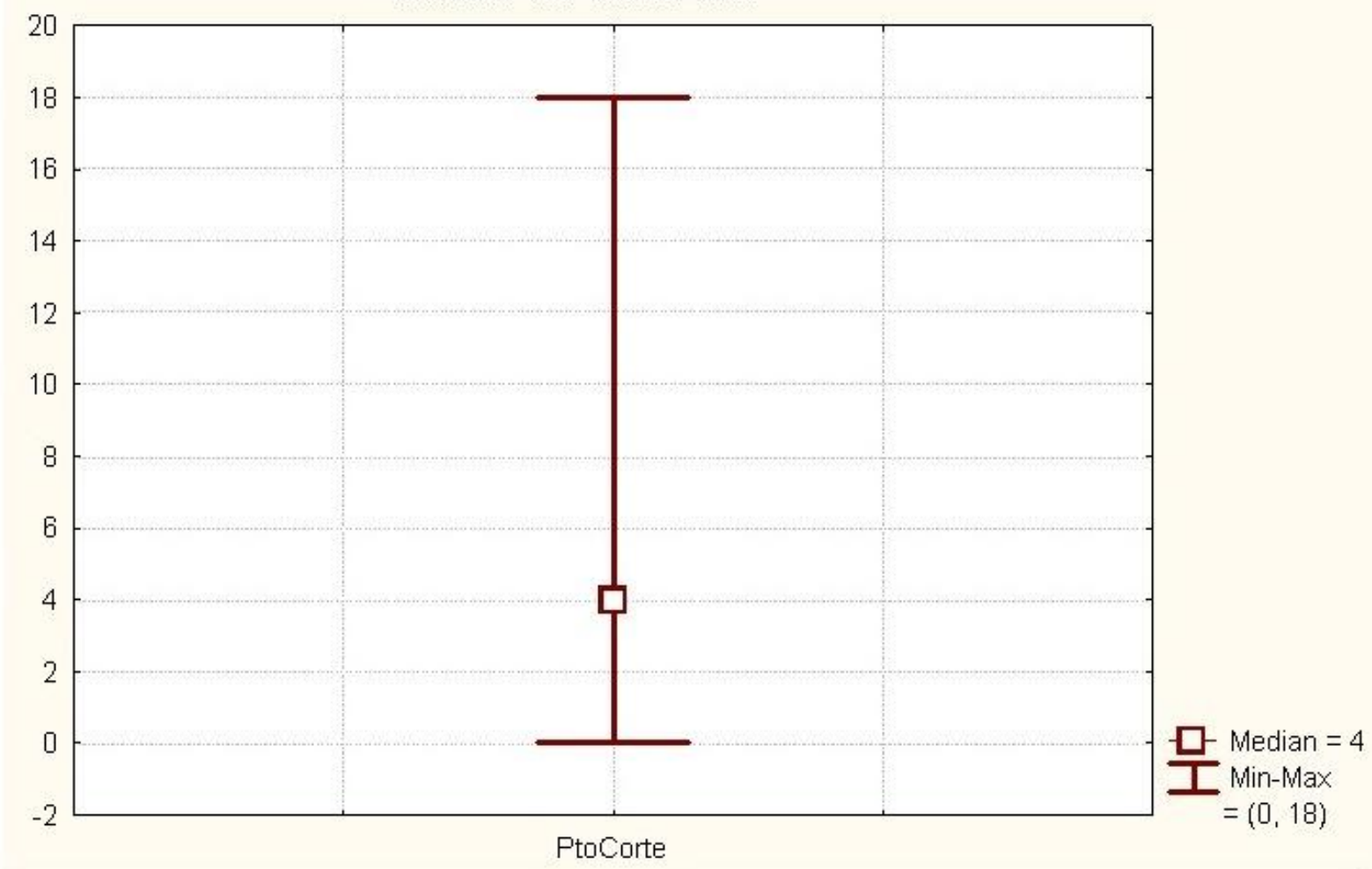

Gráfico 4: Distribución de puérperas, entrevistadas en la Maternidad de un Hospital Universitario - 2009, segundo la incidencia de puntuación.

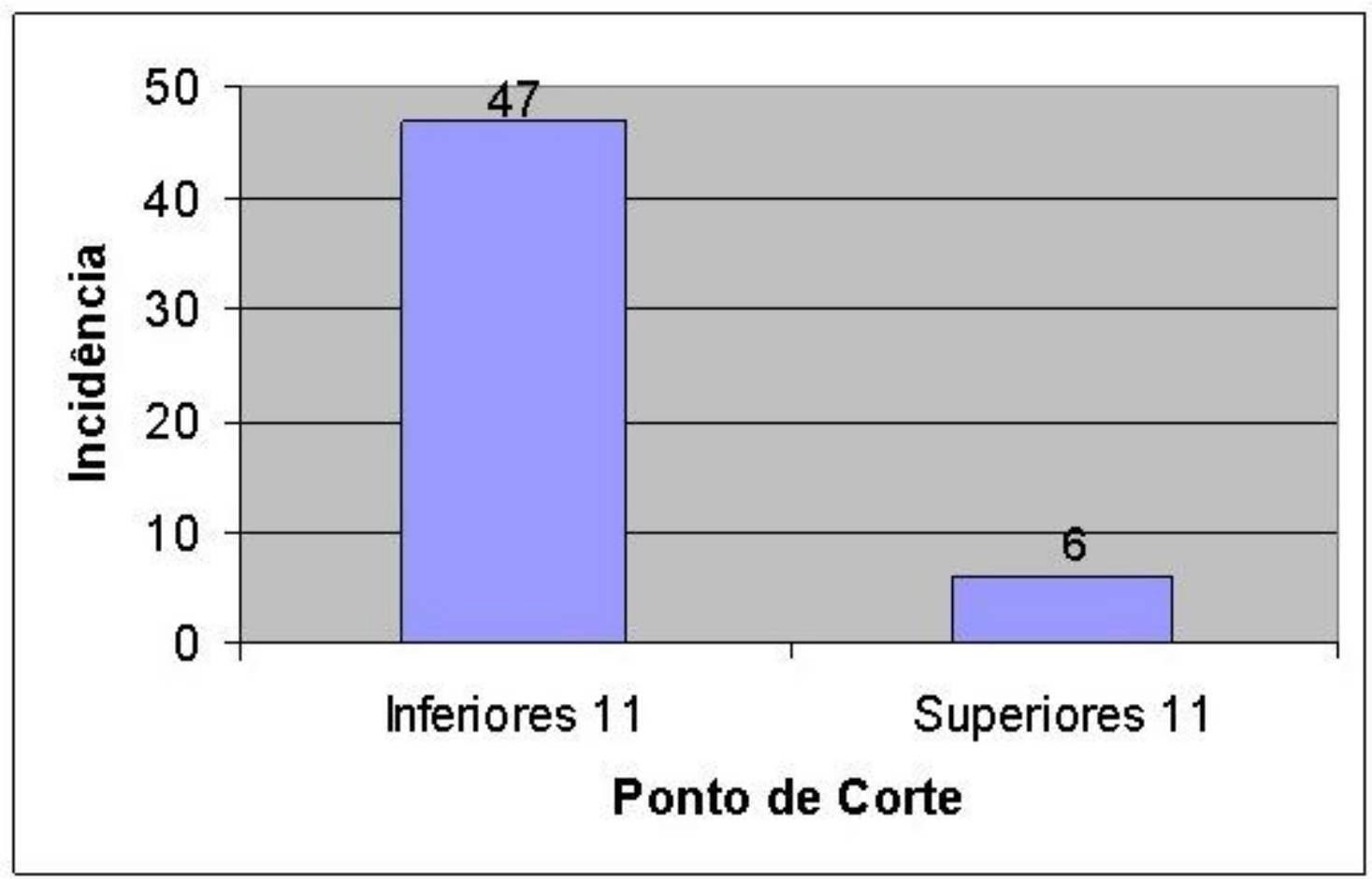


Se observó en el período comprendido por el estudio, una frecuencia de riesgo de depresión, apurada en el segundo o tercero día después del parto de $11 \%(\mathrm{n}=53)$, utilizándose como criterio de diagnóstico el valor de la EPDS $\geq 11$. Ya, un estudio realizado en São Paulo, con el mismo instrumento en segundo o tercero día del puerperio observó $18 \%(n=133)$ de las mujeres con marcadores 10 o más, en esta fase, presentaron significativa frecuencia de riesgo para DPP ${ }^{6}$. Otra investigación conducida en Brasil, en Espírito Santo, consideró el indicador mayor de 12 de la EPDS como el de mayor adecuación para el diagnóstico de DPP, la prevalencia de DPP mencionada fue de 39,4\% $(n=292)$ en mujeres que se encontraban entre 31 e 180 días después del parto ${ }^{7}$.

Las variaciones entre los índices de prevalencia de la DPP divergen entre los estudios realizados al respecto de este asunto. Los criterios diagnósticos utilizados son distintos, el período de evaluación, la población involucrada, los factores económicos, culturales y el punto de corte utilizado por las diferentes escalas favorecen tasas desiguales en relación a los valores predominantes de la depresión puerperal.

El porcentaje de mujeres con riesgo en nuestro estudio fue menor, comparado con otras investigaciones, lo que tal vez pueda ser explicado por las diferencias metodológicas entre los estudios. La primera de esas diferencias se refiere al instrumento utilizado. En Pelotas fue realizado un estudio para evaluar la prevalencia y los factores asociados a la depresión puerperal y la prevalencia encontrada fue de $19,1 \% \quad(n=410)$, mas utilizaron como instrumento la Escala de Hamilton (HAM-D), con el objetivo de medir y caracterizar la presencia de síntomas depresivos ${ }^{8}$. Además de eso, otra posible causa de la diferencia es el momento de la colecta y el tamaño de la muestra. El presente estudio utilizó el puerperio inmediato y los demás trabajos utilizaron un largo espectro de semanas para la evaluación.

En estudio realizado en el Estado de São Paulo observaron prevalencia de $37,1 \%$ de significante riesgo para depresión, entre 12 y 16 semanas de puerperio, con puntuación en la EPDS superior a $11(n=70)^{9}$. Otro trabajo brasileño, en Paraíba, encontró prevalencia semejante: $33 \%(n=84)$ de madres puérperas con sintomatología de depresión, entre 15 a 90 días de posparto, presentando puntuaciones iguales o arriba de 11 puntos en la Escala de Edinburgh ${ }^{10}$.

Atendiendo la posibilidad de identificar temprano mujeres con riesgo de desarrollar DPP o que ya se muestren deprimidas, el estudio propuesto aplica el cuestionario después del parto, cuando la paciente aun se encuentra en la maternidad, permitiendo la intervención primaria o secundaria rápida, procurando minimizar las consecuencias de la enfermedad.

Comparando las pacientes y las variables maternas (edad, número de gestaciones y tipo de parto), fue posible percibir algunas diferencias significativas entre las comparaciones. Siendo que, las puérperas entrevistadas fueron clasificadas en dos grupos, en la EPDS: las que obtuvieran puntuación $<11$ serian del grupo control (sin riesgo); y las con puntuación $\geq 11$ del grupo caso (con riesgo) para desarrollar la depresión pos-parto. En el grupo sin riesgo predominó mujeres adultas (72\%), primíparas (47\%) y que fueron sometidos a cesárea (58\%) (Tabla 1). 
Tabla 1- Variables de acuerdo con la puntuación en la escala de Edinburgh, observadas en puérperas de la Maternidad del Hospital Universitario HU/FURG- 2009.

\begin{tabular}{|c|c|c|c|c|c|}
\hline \multirow{2}{*}{\multicolumn{2}{|c|}{ VARIAVÉIS }} & \multicolumn{4}{|c|}{ ESCALA DE EDINBURGH } \\
\hline & & \multicolumn{2}{|c|}{$\begin{array}{l}<11(\mathrm{n}=47) \\
\text { Grupo controle } \\
\text { (sem risco) }\end{array}$} & \multicolumn{2}{|c|}{$\begin{array}{l}\geq 11(\mathrm{n}=6) \\
\text { Grupo caso } \\
\text { (com risco) }\end{array}$} \\
\hline \multirow{3}{*}{$\begin{array}{c}\text { DADE } \\
\text { MATERNA }\end{array}$} & & $\mathrm{n}$ & $\%$ & $\mathrm{n}$ & $\%$ \\
\hline & $10-19$ & 9 & 17 & 2 & 4 \\
\hline & $\geq 20$ anos & 38 & 72 & 4 & 7 \\
\hline \multirow{3}{*}{$\begin{array}{l}\text { NÚMERO DE } \\
\text { GESTAÇÕES }\end{array}$} & Primípara & 25 & 47 & 1 & 2 \\
\hline & Multípara & 22 & 41,5 & 5 & 9 \\
\hline & & & & & \\
\hline \multirow{2}{*}{$\begin{array}{l}\text { TIPO DE } \\
\text { PARTO }\end{array}$} & Cesária & 31 & 58 & 5 & 9 \\
\hline & Normal & 16 & 30 & 1 & 2 \\
\hline
\end{tabular}

En el grupo de riesgo predominó mujeres adultas (7\%), multíparas (9\%) y que fueran sometidas a cesárea (9\%). Por tanto, observamos la asociación entre los dos grupos en cuanto a la edad y tipo de parto, o sea, predominaron mujeres que fueron sometidas a cesárea y adulta. Sin embargo, en cuanto al número de gestaciones prevaleció en el grupo control, las primíparas y en el grupo caso, las multíparas.

Relacionando estos datos con los factores de riesgo que vienen siendo estudiados y correlacionados con la DPP, se percibe que el tipo de parto fue confirmado en el presente estudio, pues las mujeres que se sometieron a cesárea mostraron mayor riesgo para desarrollar la DPP. Hecho relevante, pues el periódico BBC Brasil.com divulgó que la cesárea perjudica la relación afectiva entre madre y niño; y las madres que hacen parto natural responde más al llanto y manifestaciones del niño ${ }^{11}$.

En lo que se refiere al número de gestaciones, el estudio evidenció mayor ocurrencia de multíparas con susceptibilidad para desarrollar la DPP. Contrariando, sin embargo, autores consideran que las primíparas tendrían mayor riesgo para desencadenar la enfermedad ${ }^{12-13}$.

Un estudio relató que, en las adolescentes, la depresión es dos veces más grave, que en las gestantes adultas, teniendo en cuenta la inmadurez emocional y la falta de seguridad en una relación con el compañero ${ }^{9}$. Sin embargo, en el presente estudio fueron las mujeres adultas las que se manifestaron con incidencia significativa para desencadenar la DPP.

\section{CONSIDERACIONES FINALES}

Al final de este estudio fue posible alcanzar el objetivo, a través de la aplicación del cuestionario, basado en la Escala de Depresión Pos-Parto de Edimburgo (EPDS), el cual es un instrumento de auto-evaluación para rastrear la depresión después la gestación, con las puérperas internadas en la maternidad del Hospital Universitario de la región sur do país.

A través de la utilización de la escala EPDS fue posible identificar la frecuencia de riesgo (11\%) para la depresión posparto en las puérperas de la maternidad, confirmando la 
importancia de la utilización de ese instrumento como ayuda para la detección precoz de la depresión puerperal y también como facilitador para el planeamiento de los cuidados prestados a la puérpera, al niño y a la familia. Lo que nos permite realizar tal afirmación es que, en el período de un mes fueran constatadas seis mujeres con probable diagnóstico de DPP, durante un año significaría la incidencia de la enfermedad en aproximadamente 72 puérperas. Además de eso, la mediana de edad fue de 26 años, significando que $50 \%$ de las mujeres se encontraban en edad fértil.

La aplicación de la EPDS evidenció ser un instrumento simple, de fácil aplicación y rápido, en lo que se refiere a la operacionalización de la etapa de colecta de datos, no exigiendo más de 10 minutos para su rellenado, el cual lo torna ideal para ser incluido en la rutina clínica de los profesionales no-especializados en el área de salud mental, con la finalidad de hacer un seguimiento de madres que presenten señales y síntomas depresivos, contribuyendo con los servicios de contra-referencia específicos, pues aminora la probabilidad de aumentar aún más su demanda.

La depresión posparto no ocurre necesariamente después del parto, puede ocurrir hasta un año después del nacimiento del niño, exigiendo un cuidado apropiado y eficaz, dirigido a la atención de las necesidades individuales y colectivas, y las particularidades del período posparto. Este estudio permitió conocer y comprender la importancia de las acciones preventivas, de la identificación precoz, del dirigirla al profesional especializado para realización del tratamiento adecuado, a fin de evitar las consecuencias devastadoras de la enfermedad para la puérpera, para el niño y su familia, mostrando que la EPDS puede convertirse en una importante herramienta para la realización de un diagnóstico más preciso y precoz de los señales y síntomas de la depresión puerperal.

La aplicación de un cuestionario cerrado de auto-evaluación de los señales y síntomas presentados por la puérpera después del parto, los cuales pueden significar una probable depresión posparto se torna pertinente teniendo en cuenta que esta enfermedad es considerada como un problema de salud pública, teniendo una incidencia de aproximadamente 10 a $20 \%$ en nuestro país. En el hospital universitario estudiado, donde no existe un instrumento específico para identificar las puérperas que presentan riesgo para desencadenar este trastorno, la EPDS facilitaría la evaluación clínica y confirmación del diagnóstico de la DPP.

\section{REFERENCIAS}

1. Pearlstein T, Howard M, Salisbury A, Zlotnick C. Postpartum depression. American Journal of Obstetrics and Gynecology. 2009; 200 (4): 357-364.

2. Clark G. - Discussing emotional health in pregnancy: the Edinburgh Postnatal Depression Scale. Br J Community Nurs. 2000; 5 (2): 91-98.

3. Mosack V, Shore ER. Screening for depression among and postpartum women. J. Community Health Nurs. 2006; 23(1): 37-47.

4. Lowdermilk DL, Perry SE, Bobak IM. O cuidado em enfermagem materna. Porto Alegre: Artmed; 20025. Figueira P, Corrêa H, Malloy-Diniz L, Romano-Silva MA. Escala de Depressão Pós-natal de Edimburgo para triagem no sistema público de saúde. Rev. Saúde Pública. 2009; 43(1): 79-84.

6. Mattar R. et al. A violência doméstica como indicador de risco no rastreamento da depressão pós-parto. Rev Bras Ginecol Obstet. 2007; 29: 470-477. 
7. Ruschi GEC, Sun SY, Mattar R, Filho AC, Zandonade E, De Lima VJ. Aspectos epidemiológicos da depressão pós-parto em amostra brasileira. Rev Psiquiatr. 2007; 29: 274-280.

8. Moraes IGS, Pinheiro RT, Da Silva RA, Horta BL, Sousa PLR, Faria AD. Prevalência da depressão pós-parto e fatores associados. Rev Saude Publica. 2006; 40: 75-70.

9. Cruz EBS, Simões GL, Cury AF. Rastreamento da depressão pós-parto em mulheres atendidas pelo Programa de Saúde da Família. Rev Bras Ginecol Obstet.2005; 27: 181-188.

10. Saraiva ERA, Coutinho MPL. A estrutura das representações sociais das mães puérperas acerca da depressão pós-parto. Rev PsicoUSF. 2007; 12: 319- 326.

11. BBC Brasil. Cesariana prejudica relação afetiva entre mãe e bebê. Jornal BBC Brasil.com. [internet]. 2008 Set. [acesso em 4 jun. 2009]. Disponível em: http://www.bbc.co.uk/portuguese/reporterbbc/story/2008/09/080904_bebe_parto_dg.shtml 12.Camacho RS, Cantinelli FC, Ribeiro CS et al. Transtornos psiquiátricos na gestação e no puerpério: classificação, diagnóstico e tratamento. Revista de Psiquiatria Clínica. 2006; 33(2): 92-102.

13. Camargo LA, Silva GA, Otta E. Mãe adolescente e solteira: vulnerabilidade no desenvolvimento de depressão pós-parto? In: 15오 Simpósio Internacional de Iniciação Científica da USP. São Paulo: 15 SIICUSP/ Videolar S.A., 2007 


\section{ESCALA DE EDINBURGH DE DEPRESIÓN POS-NATAL}

Como você recentemente teve um bebê, gostaria de saber como você está se sentindo nos últimos dias. Por favor, marque com um $\mathrm{X}$ a resposta mais próxima ao que você tem sentido.

1. Eu tenho sido capaz de rir e achar graça das coisas:

$\square$ 0-Como eu sempre fiz

$\square$ 1- Não tanto quanto antes

$\square$ 2- Sem dúvida, menos que antes

$\square$ 3- De jeito nenhum
6. Eu tenho me sentido esmagada pelas tarefas e acontecimentos do meu dia-a-dia $\square$ 3- Sim. Na maioria das vezes eu não consigo lidar bem com eles

$\square$ 2- Sim. Algumas vezes não consigo lidar bem como antes

$\square$ 1- Não. Na maioria das vezes consigo lidar bem com eles

$\square$ 0- Não. Eu consigo lidar com eles tão bem quanto antes

2. Eu sinto prazer quando penso no que está por acontecer em meu dia-a-dia

$\square 0$ - Como sempre senti

$\square$ 1- Talvez, menos que antes

$\square$ 2- Com certeza menos

$\square$ 3- De jeito nenhum

3. Eu tenho me culpado sem necessidade quando as coisas saem erradas *

$\square$ 3- Sim, na maioria das vezes

$\square$ 2- Sim, algumas vezes

1 - Não muitas vezes

$\square$ 0- Não, nenhuma vez

4. Eu tenho me sentido ansiosa ou preocupada sem uma boa razão

$\square$ 0- Não, de maneira alguma

$\square$ 1- Pouquíssimas vezes

2- Sim, algumas vezes

3- Sim, muitas vezes

5. Eu tenho me sentido assustada ou em pânico sem um bom motivo *

$\square$ 3- Sim, muitas vezes

$\square$ 2- Sim, algumas vezes

$\square$ 1- Não muitas vezes

$\square$ 0- Não, nenhuma vez
7. Eu tenho me sentido tão infeliz que eu tenho tido dificuldade de dormir *

$\square$ 3- Sim, na maioria das vezes

$\square$ 2- Sim, algumas vezes

$\square$ 1- Não muitas vezes

$\square$ 0- Não, nenhuma vez

8. Eu tenho me sentido triste ou arrasada *

$\square$ 3- Sim, na maioria das vezes

$\square$ 1- Não muitas vezes

$\square$ 0- Não, de jeito nenhum

9. Eu tenho me sentido tão infeliz que eu tenho chorado *

$\square$ 3- Sim, quase todo o tempo

$\square$ 2- Sim, muitas vezes

$\square$ 1- De vez em quando

$\square 0$ - Não, nenhuma vez

10. A idéia de fazer mal a mim mesma passou por minha cabeça *

$\square$ 3- Sim, muitas vezes, ultimamente

$\square$ 2- Algumas vezes nos últimos dias

$\square$ 1- Pouquíssimas vezes, ultimamente

$\square$ 0- Nenhuma vez $\square$ 2- Sim, muitas vezes

Fuente: MATTAR, R. et al. A violência doméstica como indicador de risco no rastreamento da depressão pós-parto. Revista Brasileira de Ginecologia e Obstetrícia, Rio de Janeiro, v.29, n.9, p.470-477, 29 set. 2007. Disponível em: <http://www.scielo.br/pdf/rbgo/v29n9/06.pdf >. Adaptado de: LOWDERMILK, D. L.; PERRY, S.E.; BOBAK, I.M. O cuidado em enfermagem materna. 5ª. ed., Porto Alegre: Artmed, 2002, 768 p. 